\title{
SSC radiation in BL Lacertae sources, the end of the tether ${ }^{\star}$
}

\author{
A. Paggi ${ }^{1}$, F. Massaro ${ }^{2}$, V. Vittorini ${ }^{3}$, A. Cavaliere ${ }^{1}$, F. D’Ammando ${ }^{1,3}$, F. Vagnetti ${ }^{1}$, and M. Tavani ${ }^{1,3}$ \\ 1 Dipartimento di Fisica, Università di Roma Tor Vergata, Via della Ricerca Scientifica 1, 00133 Roma, Italy \\ e-mail: alessandro.paggi@roma2.infn.it \\ 2 Harvard, Smithsonian Astrophysical Observatory, 60 Garden Street, Cambridge, MA 02138, USA \\ 3 INAF, via Fosso del Cavaliere 1, 00100 Roma, Italy
}

Received 31 March 2009 / Accepted 12 July 2009

\begin{abstract}
Context. The synchrotron-self Compton (SSC) radiation process is widely held to provide a close representation of the double peaked spectral energy distributions from BL Lac Objects (BL Lacs). This subclass of Active Galactic Nuclei is marked by non-thermal beamed radiations, highly variable on timescales of days or less. Their outbursts in the $\gamma$ rays relative to the optical/X rays might be surmised to be enhanced in BL Lacs as these photons are upscattered via the inverse Compton (IC) process.

Aims. From the observed correlations among the spectral parameters (peak frequencies, fluxes and curvature) during optical/X-ray variations we aim at predicting corresponding correlations in the $\gamma$-ray band, and the actual relations between the $\gamma$-ray and the X-ray variability consistent with the SSC emission process.

Methods. We start from the homogeneous single-zone SSC source model, with log-parabolic energies distributions of emitting electron as required by the X-ray data of many sources. We find relations among spectral parameters of the IC radiation in both the Thomson (for Low energy BL Lacs) and the Klein-Nishina regimes (mainly for High energy BL Lacs); whence we compute how variability is driven by a smooth increase of key source parameters, primarily the root mean square electron energy.

Results. In the Klein-Nishina regime the model predicts for HBLs lower inverse Compton fluxes relative to synchrotron, and milder $\gamma$-ray relative to X-ray variations. Stronger $\gamma$-ray flares observed in some HBLs like Mrk 501 are understood in terms of additional, smooth increases also of the emitting electron density. However, episodes of rapid flares as recently reported at TeV energies are beyond the reach of the single component SSC model with one dominant varying parameter. Furthermore, spectral correlations at variance with our predictions, as well as $\mathrm{TeV}$ emissions in LBL objects (like BL Lacertae itself) cannot be explained in terms of the simple HSZ SSC model, and in these cases the source may require additional electron populations in more elaborate structures like decelerated relativistic outflows or sub-jet scenarios.

Conclusions. We provide a comprehensive benchmark to straightforwardly gauge the capabilities and effectiveness of the SSC radiation process. The single component SSC source model in the Thomson regime turns out to be adequate for many LBL sources. In the mild Klein-Nishina regime it covers HBL sources undergoing variations driven by smooth increase of a number of source parameters. However, the simple model meets its limits with the fast/strong flares recently reported for a few sources in the TeV range; these clearly require sudden accelerations of emitting electrons in a second source component.
\end{abstract}

Key words. galaxies: active - galaxies: jets - BL Lacertae objects: general - radiation mechanisms: non-thermal

\section{Introduction}

Blazars are among the brightest Active Galactic Nuclei (AGNs) observed, with inferred (isotropic) luminosities up to $L \sim$ $10^{47} \mathrm{erg} \mathrm{s}^{-1}$. Actually these sources are widely held to be AGNs radiating from a relativistic jet closely aligned to the observer line of sight; this emits non-thermal radiations with observed fluxes enhanced by relativistic effects so as to often overwhelm other, thermal or reprocessed emissions.

BL Lacs in particular are Blazars showing no or just weak emission lines. Their spectra may be represented as a continuous spectral energy distribution (SED) $S_{v}=v F_{v}$ marked by two peaks: a lower frequency one, widely interpreted as synchrotron emission by highly relativistic electrons; and a higher frequency counterpart, believed to be inverse Compton upscattering by the same electrons of seed photons either emitted by an external source (external Compton, EC, see e.g. Sikora et al. 1994), or provided by the photons of the very

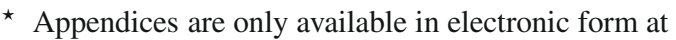
http://www. aanda.org
}

synchrotron radiation (synchrotron-self Compton, SSC; see, e.g., Marscher \& Gear 1985; Maraschi et al. 1992).

The BL Lacs are usually classified in terms of the frequency of their synchrotron peak (see Padovani \& Giommi 1995): for the low frequency BL Lacs (LBLs) the peak lies in the infraredoptical bands, while the inverse Compton (IC) component peaks at MeV energies; instead, high frequency BL Lacs (HBLs) feature a first peak in the X-ray band and the second one at $100 \mathrm{GeV}$ energies or beyond; intermediate frequency BL Lacs (IBLs) stand in the middle, with the first peak at optical frequencies and the second peak at $\sim 1 \mathrm{GeV}$.

BL Lac Objects also show rapid variability on timescales of days or shorter, during which the sources undergo strong flux variations often named "flares".

The homogeneous single zone (HSZ) SSC model (based, in particular, on a single electron population) constitutes an attractively simple source structure worth to be extensively tested and pushed to its very limits, as we discuss below; following Katarzyński et al. (2005), Tramacere et al. (2007), we focus on deriving the simultaneous changes of the synchrotron and IC fluxes of BL Lacs to be expected from an updated version 
of SSC. We use a realistic log-parabolic energy distribution of the electrons radiating the synchrotron photons and upscattering them by IC in either the Thomson or the Klein Nishina (KN) regime.

\section{Background relations}

\subsection{Log-parabolic electron distribution and spectra}

The SEDs of BL Lacs exhibit two distinct peaks or humps. To describe these in detail a number of spectral models have been used over the years: power-laws with an exponential cutoff, broken power-laws, or spectra curved in the shape of a log-parabola. The latter are being increasingly used, as they generally provide more accurate fits than broken power-laws in the optical X-ray bands, with residuals uniformly low throughout a wide energy range (see discussion by Landau et al. 1986; Tanihata et al. 2004; Massaro et al. 2004a; Tramacere et al. 2007; Nieppola et al. 2006; Donnarumma et al. 2009) ${ }^{1}$.

So we adopt log-parabolae in view of their optimal performance as a precise fitting tool. Moreover, they are also effective in yielding simple, analytical expressions (given in their exact form in Appendix A, following Eqs. (A.6), (A.9) and (A.15)) for peak frequencies and fluxes in both in the inverse Compton regimes, Thomson and Klein-Nishina. Finally, the physically interesting feature of log-parabolic spectra is their direct link to the acceleration processes of the emitting particles; in fact, as shown in Appendix B, log-parabolic emitted spectra simply relate to log-parabolic particle energy distributions, that in turn naturally arise from systematic and stochastic acceleration processes as dealt with by the Fokker-Planck equation.

Thus for the synchrotron SED we write

$S_{v}^{\mathrm{s}}=S_{0}^{\mathrm{s}}\left(\frac{v}{v_{0}}\right)^{-\left(a_{\mathrm{s}}-1\right)-b_{\mathrm{s}} \log \left(\frac{v}{v_{0}}\right)} ;$

here $a_{\mathrm{s}}$ gives the constant component of the spectral index for the energy flux and $b_{\mathrm{s}}$ is the spectral curvature. In the following we shall denote with $\xi$ the frequency where the SED peaks and by $S$ the related flux. Spectral curvature values are observed to be around 0.2 (Massaro et al. 2004b) and decreasing with time and/or frequency as given by Eqs. (B.11) and (B.13) of Appendix A; since the decrease is mild during flares (which are essentially dominated by systematic acceleration processes), we can consider a nearly constant spectral curvature to a first approximation. Then one finds the scaling relations $\xi \propto B \gamma_{\mathrm{p}}^{2} \delta$ and $S \propto B^{2} \gamma_{\mathrm{p}}^{2} n R^{3} \delta^{4}$, where $n$ is the particle density, $R$ is the emitting region radius, $B$ is the magnetic field, $\delta$ is the beaming factor, and $\gamma_{\mathrm{p}} m c^{2}$ is the square root of the mean quadratic energy (thereafter rms energy) of the particle distribution.

An analogous expression holds for the inverse Compton radiation

$S_{v}^{\mathrm{c}} \approx S_{0}^{\mathrm{c}}\left(\frac{v}{\hat{v}_{0}}\right)^{-\left(a_{\mathrm{c}}-1\right)-b_{\mathrm{c}} \log \left(\frac{v}{\hat{v}_{0}}\right)}$,

which in the Thomson regime the IC has less curved spectra with $b_{\mathrm{c}} \approx b_{\mathrm{s}} / 2$. At nearly constant curvature the parameters to

\footnotetext{
1 The log-parabolic particle energy distribution could be made asymmetric by adding a powerlaw tail at low energies (see Massaro et al. 2006) to represent also low frequency data, e.g., radio in addition to optical/X-ray data; however, here we will focus on the SEDs around their peaks and so we do not use such additions.
}

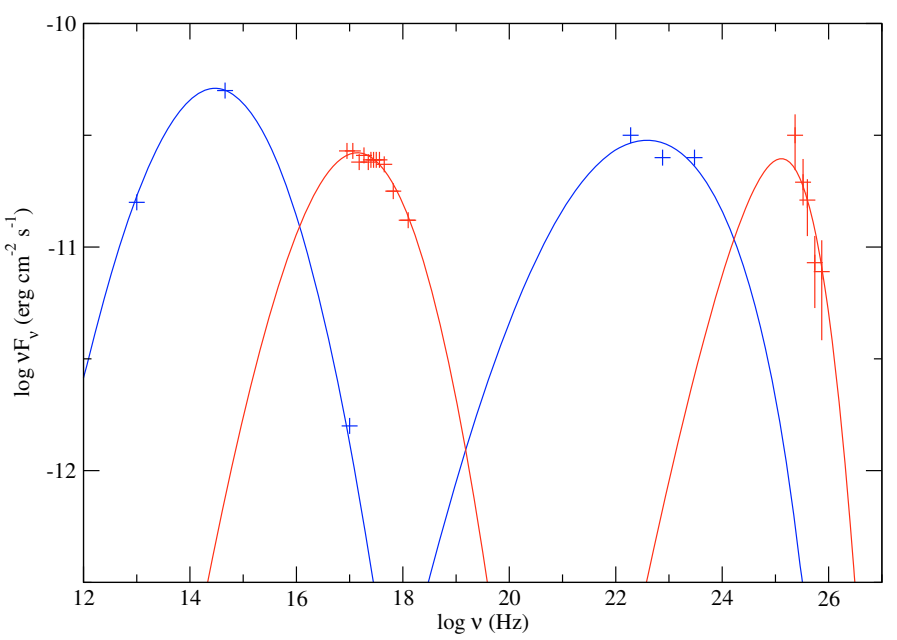

Fig. 1. Two examples of exact, steady SEDs provided by numerical simulations (code by Massaro 2007) after the HSZ SSC model from relativistic electrons with a log-parabolic energy distribution $N(\gamma) \propto$ $\gamma^{-s-r \log \gamma}$ (see Appendix B). The two characteristic humps correspond to synchrotron emission (left) and to inverse Compton scattering (right), either in the Thomson regime (blue line) or in the KN regime (red line). Standard sources that are so fitted include the low state of $0716+714$ (an IBL, blue crosses) observations of November 1996 by BeppoSAX, AIT and EGRET (Tagliaferri et al. 2003); and 1ES 1553+113 (a HBL, red crosses) observations of April 2005 by Swift and April-May 2005 by MAGIC (Massaro 2007).

focus on are $\epsilon \propto B \gamma_{\mathrm{p}}^{4} \delta$ and $C \propto B^{2} \gamma_{\mathrm{p}}^{4} n^{2} R^{4} \delta^{4}$ for the SED peak frequency and flux, respectively. In the extreme Klein-Nishina regime we obtain instead more curved spectra with $b_{\mathrm{c}} \approx 5 b_{\mathrm{s}}$, and $\epsilon \propto \gamma_{\mathrm{p}} \delta$ and $C \propto B n^{2} R^{4} \delta^{4}$ hold (see Appendix A for more details, and massaro2008a,b).

Such spectra are radiated by electron populations with a log-parabolic energy distribution, namely

$N(\gamma)=N_{0}\left(\frac{\gamma}{\gamma_{0}}\right)^{-s-r \log \left(\frac{\gamma}{\gamma_{0}}\right)}$.

The result is easily inferred and straightforward to compute on using the delta-function approximations for the single particle emissions (see Appendix A); it is found to hold also for the exact shape of the latter with the numerical simulations outlined in Fig. 1, and reported in full detail in Figs. B.1 and B.2.

\subsection{Source parameters}

Using the previous scalings, with the relation $R=c \Delta t \delta /(1+z)$ between the observed variation time $\Delta t$ and the size $R$ of the emitting region (assuming spherical symmetry in the rest frame), we obtain relations of source parameters with the observables. In the Thomson regime we find

$$
\begin{aligned}
\gamma_{\mathrm{p}} & \propto \epsilon^{\frac{1}{2}} \xi^{-\frac{1}{2}} \\
R & \propto \epsilon^{\frac{1}{2}} S^{\frac{1}{2}} \Delta t^{\frac{1}{2}} \xi^{-1} C^{-\frac{1}{4}} \\
B & \propto \xi^{3} C^{\frac{1}{4}} \Delta t^{\frac{1}{2}} \epsilon^{-\frac{3}{2}} S^{-\frac{1}{2}} \\
\delta & \propto \epsilon^{\frac{1}{2}} S^{\frac{1}{2}} \xi^{-1} C^{-\frac{1}{4}} \Delta t^{-\frac{1}{2}} \\
n & \propto \xi^{2} C^{\frac{5}{4}} \epsilon^{-\frac{3}{2}} S^{-\frac{3}{2}} \Delta t^{-\frac{1}{2}} .
\end{aligned}
$$

For the extreme KN regime we find instead:

$\gamma_{\mathrm{p}} \propto \xi^{\frac{3}{5}} \epsilon^{\frac{3}{5}} C^{\frac{1}{5}} \Delta t^{\frac{2}{5}} S^{-\frac{2}{5}}$ 
Table 1. Source parameters for some BL Lac sources.

\begin{tabular}{cccccccc}
\hline \hline Source name & $z$ & $\gamma_{\mathrm{p}}$ & $R(\mathrm{~cm})$ & $B(\mathrm{G})$ & $\delta$ & $n\left(\mathrm{~cm}^{-3}\right)$ & $r$ \\
\hline $0716+714$ (low state) & 0.31 & $3.36 \times 10^{3}$ & $2.61 \times 10^{17}$ & $1.51 \times 10^{-1}$ & 10 & 0.41 & 1.18 \\
$0716+714$ (high state) &, & $9.92 \times 10^{3}$ & $7.79 \times 10^{17}$ & $7.64 \times 10^{-2}$ & 10 & 0.03 & 1.18 \\
1ES 1553+113 & 0.25 & $3.39 \times 10^{4}$ & $0.64 \times 10^{16}$ & $9.80 \times 10^{-1}$ & 10 & 2.04 & 1.49 \\
Mrk 501(low state) & 0.034 & $1.88 \times 10^{5}$ & $0.38 \times 10^{16}$ & $9.29 \times 10^{-2}$ & 15 & 0.62 & 0.81 \\
Mrk 501(high state) &, & $2.58 \times 10^{5}$ & $0.59 \times 10^{15}$ & $3.85 \times 10^{-1}$ & 15 & 23.49 & 0.74 \\
\hline
\end{tabular}

$$
\begin{aligned}
& R \propto \epsilon^{\frac{2}{5}} S^{\frac{2}{5}} \Delta t^{\frac{3}{5}} \xi^{-\frac{3}{5}} C^{-\frac{1}{5}} \\
& B \propto \xi^{\frac{2}{5}} S^{\frac{2}{5}} \epsilon^{-\frac{8}{5}} C^{-\frac{1}{5}} \Delta t^{-\frac{2}{5}} \\
& \delta \propto \epsilon^{\frac{2}{5}} S^{\frac{2}{5}} \xi^{-\frac{3}{5}} C^{-\frac{1}{5}} \Delta t^{-\frac{2}{5}} \\
& n \propto \xi^{\frac{11}{5}} C^{\frac{7}{5}} \epsilon^{-\frac{4}{5}} S^{-\frac{9}{5}} \Delta t^{-\frac{1}{5}} .
\end{aligned}
$$

Note that a delay of the order of $\Delta t$ may elapse between synchrotron and IC spectral variations.

Numerical values for the prefactors are given in Appendix C, where normalizations are chosen referring to a typical LBL source with $S \approx 10^{-11} \mathrm{erg} \mathrm{cm}^{-2} \mathrm{~s}^{-1}, \xi \approx 10^{14} \mathrm{~Hz}, C \approx$ $10^{-11} \mathrm{erg} \mathrm{cm}^{-2} \mathrm{~s}^{-1}, \epsilon \approx 10^{22} \mathrm{~Hz}$; and to a typical $\mathrm{HBL}$ source with $S \approx 10^{-10} \mathrm{erg} \mathrm{cm}^{-2} \mathrm{~s}^{-1}, \xi \approx 10^{18} \mathrm{~Hz}, C \approx$

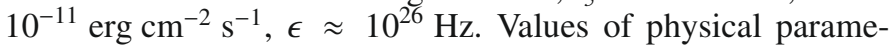
ters for some sources are given in Table 1. Note that the simple Eqs. (4)-(8) provide parameter values in close agreement with the ones needed to fit the observations of LBL or IBL sources in Thomson regime; on the other hand the extreme $\mathrm{KN}$ limit underlying Eqs. (9)-(13) would yield disagreement for HBLs, indicating that these sources just border the $\mathrm{KN}$ regime.

We evaluate the position $\xi_{\mathrm{T}}$ of the synchrotron peak when the transition between the two IC regimes occurs, to find

$\xi_{\mathrm{T}} \approx 7.15 \times 10^{15}\left(\frac{B}{0.1 \mathrm{G}}\right)^{\frac{1}{3}}\left(\frac{\delta}{10}\right)(1+z)^{-1} \mathrm{~Hz}$,

or equivalently

$\xi_{\mathrm{T}} \approx 1.96 \times 10^{16}\left(\frac{\gamma_{\mathrm{p}}}{10^{4}}\right)^{-1} 10^{\frac{1}{2}\left(1-\frac{1}{5 b}\right)}\left(\frac{\delta}{10}\right)(1+z)^{-1} \mathrm{~Hz}$.

So we conclude that in LBLs, which feature the synchrotron peak at optical/IR frequencies with $\xi \approx 10^{14} \mathrm{~Hz}$, the majority of photons of the IC peak are upscattered in the Thomson regime; whereas in extreme HBLs, which show the synchrotron peak in the X-ray band with $\xi \approx 10^{18} \mathrm{~Hz}$, the majority of IC peak photons are upscattered in the Klein-Nishina regime.

\subsection{Spectral correlations during $X$-ray spectral variations}

Correlations in the synchrotron emission between $S$ and $b_{\mathrm{s}}$ with $\xi$ can be used to pinpoint the main driver of the spectral changes during X-ray variations (see also Tramacere et al. 2007). In fact, the synchrotron SED peak scales (for nearly constant spectral curvature as anticipated in Sect. 2.1) as $S \propto n R^{3} \gamma_{\mathrm{p}}^{2} B^{2} \delta^{4}$ at the peak frequency itself scaling as $\xi \propto \gamma_{\mathrm{p}}^{2} B \delta$.

Thus on writing the dependence of $S$ on $\xi$ in the form of a powerlaw $S \propto \xi^{\alpha}$, we expect $\alpha=1$ to apply when the spectral changes are driven mainly by variations of the electrons rms energy; $\alpha=2$ for dominant changes of the magnetic field; $\alpha=4$ if changes in the beaming factor dominate; $\alpha=\infty$ formally applies for changes only in the number density of the emitting particles. Results by the latter authors focus on $\gamma_{\mathrm{p}}$ and $B$ as dominant drivers. Starting from such correlations we aim at predicting the expected correlations and flux variations in the $\gamma$-ray band.

Particle rms energy variations are of particular interest because they naturally arise as a consequence of systematic plus stochastic acceleration of the electron population (details in Appendix B).

\section{3. $\gamma$-ray spectra}

\subsection{Spectral correlations}

The above results lead us to expect specific correlations between SED peak frequencies and fluxes as follows. In the case of dominant rms particle energy variations we expect for the synchrotron emission

$S \propto \xi$,

while for the IC we have

$C \propto \epsilon$

for LBL objects, and

$C \approx$ const.

for extreme HBLs.

In the case of magnetic field variations we have for the synchrotron emission

$S \propto \xi^{2}$,

while for the IC we have

$C \propto \epsilon^{2}$

for LBL objects, and

$C \propto \epsilon^{\tau}$

formally with $\tau=\infty$ for extreme HBLs.

To complete the above picture, we consider how saturation affects correlations. Denoting the total number of particles with $N_{\mathrm{T}}$, in the jet frame we have for the synchrotron luminosity $L_{\mathrm{s}} \propto N_{\mathrm{T}} \gamma_{\mathrm{p}}^{2}$, while for IC luminosity we have $L_{\mathrm{C}} \propto\left(N_{\mathrm{T}} \gamma_{\mathrm{p}}^{2}\right)^{2}$ and $L_{\mathrm{C}} \propto N_{\mathrm{T}}^{2}$ in Thomson and $\mathrm{KN}$ regime, respectively. Consider the case where the total power available to the jet is limited, e.g., in the BZ mechanism (Blandford \& Znajek 1977) of power extraction from a maximally rotating black hole with an extreme, radiation pressure dominated disk that holds the pressure of the magnetic field facing the black hole horizon (see discussion by Cavaliere \& D'Elia 2002, and references therein). Then the power has an upper bound $L_{\mathrm{BZ}} \approx 2 \times 10^{45} \mathrm{M}_{9} \mathrm{erg} \mathrm{s}^{-1}$; as a consequence, we expect an effect of saturation on the SED peaks as the emitted power, which represents a substantial fraction of the total jet power (see Ghisellini et al. 2008), approaches $L_{\mathrm{BZ}}$. For 


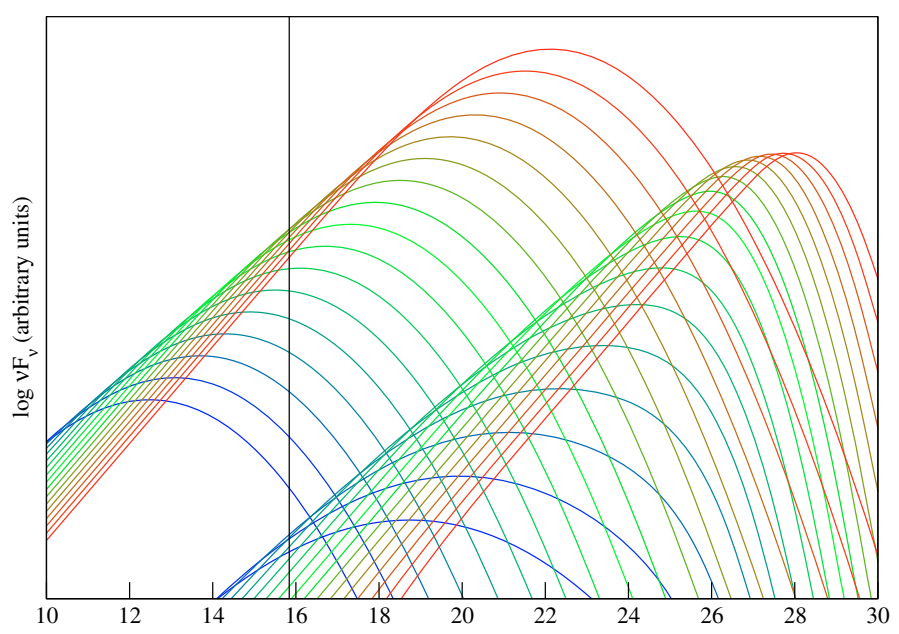

Fig. 2. To illustrate the differences of the IC SEDs in the Thomson and in the extreme $\mathrm{KN}$ regime, we show the results of numerical simulations (code by Massaro 2007) after the HSZ SSC model, for different sources with larger and larger rms energy of the radiating particles and other parameters kept constant (including the curvature of the particle energy distribution). The vertical line indicates the frequency of the synchrotron peak where the IC scattering changes over the Thomson to the $\mathrm{KN}$ regime (see Eq. (14)). Actual sources in their evolution (represented in Figs. B.1 and B.2) vary their spectral curvature somewhat and only span a limited range in frequency and flux to the left (LBLs) or to the right (HBLs) of the line, with only the IBLs likely to cross it.

the total power, including radiative, kinetic and magnetic components, $L_{\mathrm{tot}} \approx L_{\mathrm{BZ}}$ we envisage $N_{\mathrm{T}} \sim \gamma_{\mathrm{p}}^{-2}$, that is, the particles effectively accelerated to the increasing $\gamma_{\mathrm{p}}$ decrease in number on approaching the BZ limit.

The data analyses by Tramacere et al. $(2007,2009)$ for the variations of Mrk 421 are consistent with the beginning of such a saturation effect, as they show a declining value of $\alpha$ when the peak moves toward the highest energies and approaches $L \approx$ $10^{46} \mathrm{erg} \mathrm{s}^{-1}$. Partial saturation effects can also be explained in terms of systematic and stochastic acceleration processes; in particular, when the stochastic prevails over the systematic acceleration (as is bound to occur at high energies, see Appendix A) we expect $\alpha$ to decrease toward 0.6, i.e. $S \propto \xi^{0.6}$.

\subsection{Related variabilities}

We now focus on related variabilities between IC and synchrotron fluxes; here we are mainly interested on increases of peak frequencies and fluxes, as driven by acceleration processes of the emitting particles or by a growing magnetic field (see also Katarzyński et al. 2005).

For LBLs, that radiate mostly in the Thomson regime, we predict from Eqs. (16)-(21) a quadratic or linear variation of $C$ with respect to $S$, depending on the parameter that mainly drives source variations. That is to say, we expect

$\Delta \ln C \approx 2 \Delta \ln S$

in the case of dominant particle rms energy variations, or

$\Delta \ln C \approx \Delta \ln S$

for dominant magnetic field variations (see also Figs. 2 and 4).

Instead, for HBLs that radiate closer to the Klein-Nishina regime, we obtain a weaker $\gamma$-ray variability due to the decreasing $\mathrm{KN}$ cross section, leading in the extreme to

$\Delta \ln C \approx 0$ in case of dominant particle rms energy variations, or

$\Delta \ln C \approx \frac{1}{2} \Delta \ln S$

for dominant magnetic field variations. Expected correlations are summarized in Table 2.

From Figs. 2 and 4 it is seen that the transition to the $\mathrm{KN}$ regime has three effects on the IC spectrum: first, it brakes the peak frequency increase, because the energy the photon can gain is limited to the total electron energy $\gamma m c^{2}$; second, it reduces the flux increase at the SED peak, as a consequence of reduced cross section; third, it increases the spectral curvature near the peak as a consequence of frequency compression.

Viceversa, variations observed in IC section of the spectrum are related via Eqs. (22)-(25) to variations expected in the synchrotron emission; in particular, in HBLs $\gamma$-ray variations ought to have enhanced counterparts in $\mathrm{X}$ rays.

Up to now Figs. 2 and 4 may be interpreted as a collection of different sources or of unrelated states of a single source; henceforth we will focus on the interpretation in terms of evolution in a flaring source where the parameters vary in a continuous fashion; in particular, $\gamma_{\mathrm{p}}$ increases under the drive of systematic and stochastic electron accelerations, in keeping with the description in terms of a continuity or kinetic equation of the FokkerPlanck type as given in detail in Appendix B. Signatures of such an evolution are provided not only by continuous growth of the peak frequencies, but even more definitely by the irreversible decrease of the spectral curvature (in terms of time or frequency) under the drive of the stochastic acceleration, see Eq. (B.13) and the observations by Tramacere et al. (2007, 2009). In fact, for a single electron population the curvature is to slowly decrease or stay nearly constant on the timescale of the systematic acceleration. So a sudden increase of curvature signals the injection of a new population/component.

In the following we will neglect radiative cooling, which does not strongly affect the spectral shape around the peaks; such a process will be particularly relevant when the source, after flaring up, relaxes back to a lower state upon radiating away its excess energies from high frequencies downwards (see Appendix B).

\subsection{Specific sources}

We have applied the above simple expectations to two sources, 0716+714 (IBL) and Mrk 501 (HBL), among the few to date to provide extended simultaneous coverage of two different source states in $\mathrm{X}$ rays and $\gamma$ rays.

The first is widely considered to be an IBL. In the low state observed by Automatic Imaging Telescope (AIT) in November 14 1996, the synchrotron peak at about at about $10^{14.5} \mathrm{~Hz}$ falls quite below the threshold frequency expressed by Eqs. (14) or (15), so we expect for this source the IC scattering to occur mostly in the Thomson regime; simultaneous $\gamma$-ray observations were carried out by EGRET. The high activity state of the source is described by data relative to GASP project of the WEBT and AGILE-GRID observations of 2007 September 7-12 (see Fig. 6). The source has shown similarly low flux levels during EGRET observations (Tagliaferri et al. 2003); so for flares with short duty cycle, it is not unreasonable to evaluate variations between the two distant states in the absence of closer observations; curvature variation is not required to fit the data, as we expect at lower energies where systematic dominates the stochastic acceleration (see Appendix A). As shown in Figs. 3 and 5, peak flux and frequency variations of $0716+714$ appear to be in between the lines 
Table 2. Spectral correlations (for $r \simeq$ const.). We have denoted with $\left(\gamma_{\mathrm{p}}\right)$ or $(B)$ the variations driven by increases of rms electron energy or magnetic field, respectively.

\begin{tabular}{llcl}
\hline \hline Process & Peak flux and frequency & Flux-frequency correlation & $S-C$ correlation \\
\hline synchrotron & $\begin{array}{l}S \propto R^{3} B^{2} \gamma_{\mathrm{p}}^{2} n \delta^{4} \\
\xi \propto B \gamma_{\mathrm{p}}^{2} \delta\end{array}$ & $S \propto \xi^{\alpha}\left\{\begin{array}{l}\alpha=1\left(\gamma_{\mathrm{p}}\right) \\
\alpha=2(B)\end{array}\right.$ \\
\hline IC Thomson & $\begin{array}{l}C \propto R^{4} B^{2} \gamma_{\mathrm{p}}^{4} n^{2} \delta^{4} \\
\epsilon \propto B \gamma_{\mathrm{p}}^{4} \delta\end{array}$ & $C \propto \epsilon^{\alpha}\left\{\begin{array}{l}\alpha=1\left(\gamma_{\mathrm{p}}\right) \\
\alpha=2(B)\end{array}\right.$ & $\begin{array}{l}C \propto S^{2} \\
C \propto S\end{array}$ \\
\hline IC KN & $C \propto R^{4} B n^{2} \delta^{4}$ & $C \propto \epsilon^{\alpha} \begin{cases}\alpha=0\left(\gamma_{\mathrm{p}}\right) \\
\alpha=\infty(B)\end{cases}$ & $\begin{array}{l}C \approx \text { const. } \\
C \propto S^{1 / 2}\end{array}$ \\
\hline
\end{tabular}

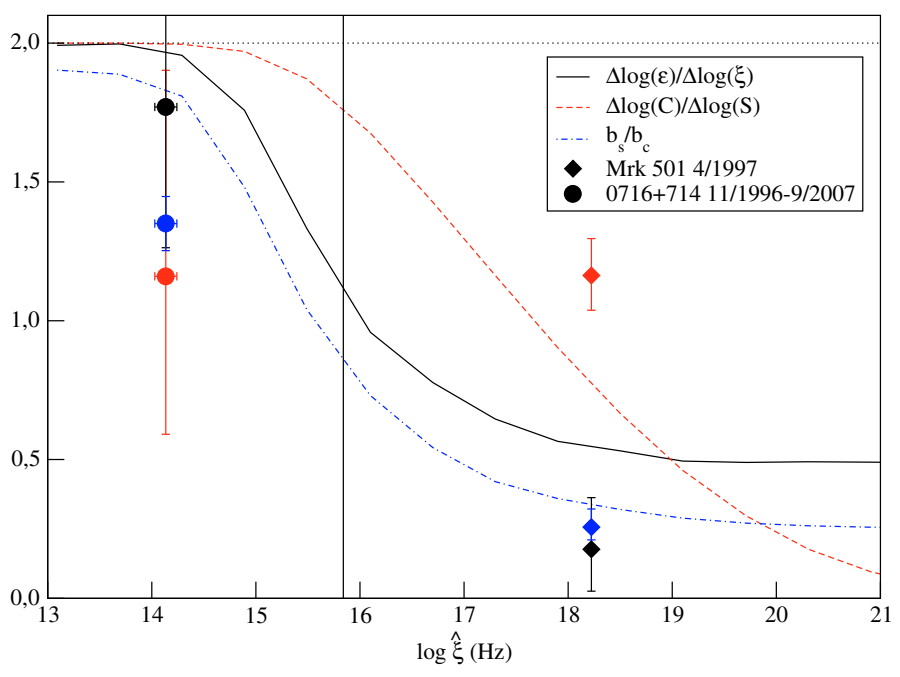

Fig. 3. Computed changes of spectral parameters corresponding to extended increase of rms particle energy with the other source parameters kept constant. As functions of $\hat{\xi}=\xi(B / 0.1 \mathrm{G})^{-1 / 3}(\delta / 10)^{-1}(1+z)$ (where $\xi$ is the synchrotron SED peak frequency, see Eq. (14)), the full line represents the ratio $\Delta \ln \epsilon / \Delta \ln \xi$, the dashed line the ratio $\Delta \ln C / \Delta \ln S$ and the dot-dashed line the curvature ratio $b_{\mathrm{s}} / b_{\mathrm{c}}$ at the peaks. Circles represent the parameters of $0716+714$ as observed on November 1996 and September 2007 (Tagliaferri et al. 2003; Chen et al. 2008), and diamonds represent Mrk 501 observations of April 1997 (Djannati-Atai et al. 1999; Massaro et al. 2006), each with the same colours as the lines. Changes of $n$ only would yield $\Delta \ln C=$ $2 \Delta \ln S$ at all frequencies, represented by the horizontal dotted line; added to a pure rms energy increase, a moderate increase of $n$ can account for the upward deviations of observed values.

representing increasing rms particle energy and increasing magnetic field, and so they may be described in terms of HSZ SSC with simultaneous variations of these two parameters.

For the HBL source Mrk 501 we consider the two states described by Massaro et al. (2006), with simultaneous BeppoSAX and CAT observations of April 7 and 16 1997. To understand the behavior of this source, from Figs. 3 and 5 it is seen that variations of $B$ alone are ineffective and require complementary $n$ increase by a factor of about 30; variations of $\gamma_{p}$ are adequate in the KN regime, and only require a complementary doubling of $n$ (which by itself would yield $\Delta \ln C \approx 2 \Delta \ln S$ in both scattering regimes) to yield higher $\gamma$-ray flux increase. Note that in going to higher energies the spectral curvature decreases somewhat (from $b=0.161 \pm 0.007$ to $b=0.148 \pm 0.005$ for the low and high state, respectively) in keeping with model predictions; such a behavior is interesting as it marks a smooth growth, if anything, in the number of emitting particles as contrasted with sudden, substantial re-injection of nearly monoenergetic electrons that

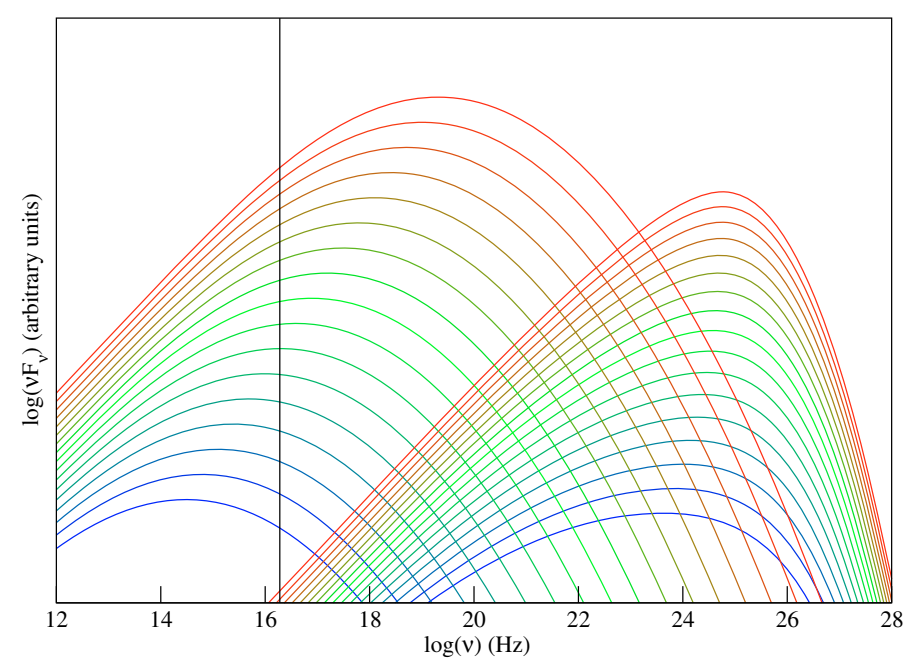

Fig. 4. As in Fig. 2, we show SEDs obtained from numerical simulations (code by Massaro 2007) of HSZ SSC radiations, for different sources with larger and larger magnetic field $B$ and other parameters kept constant. The vertical line again indicates the transition frequency from the Thomson to the $\mathrm{KN}$ regime (see Eq. (15)); as $B$ increases, the IC scattering changes its regime, and reduced cross section yields similar compression effects as in Fig. 2.

would suddenly reverse the otherwise irreversible decrease of $r$ and $b$. Such a smooth growth obtains in a scenario of an expanding blast-wave that progressively involves more electrons (see, e.g., Ostriker \& McKee 1988; Lapi et al. 2005; Vietri 2006).

We stress that in HBL sources even small flux variations in $\gamma$ rays are expected to have enhanced and observable counterparts in $\mathrm{X}$ rays. We suggest that such variations should be checked upon $\gamma$-ray "alarms", the inverse triggering relative to usual. The absence of such lower energies counterparts will indicate, for example, a flare driven dominantly by a particle number density increase associated with a magnetic field decrease $n \propto B^{-2}$, causing $S \approx$ const. but a decreasing synchrotron peak frequency; such a kind of flare may easily drown into the primary synchrotron component.

\subsection{Limiting timescales}

As shown in Appendix B, systematic and stochastic acceleration processes occur in the sources on timescales $t_{1}=1 / \lambda_{1}$ and $t_{2}=$ $1 / \lambda_{2}$, respectively. Limits to the variability timescales $\Delta t$ relate to the apparent size of the emission region by $\Delta t \geqslant R(1+z) / c \delta$ (see Sect. 2.2); rapid variations require small emitting sources, but these could be optically thick to the pair production process. In fact, $\gamma$-ray photons collide with less energetic ones to produce $\mathrm{e}^{ \pm}$pairs, and the cross section of this process tops at a value 


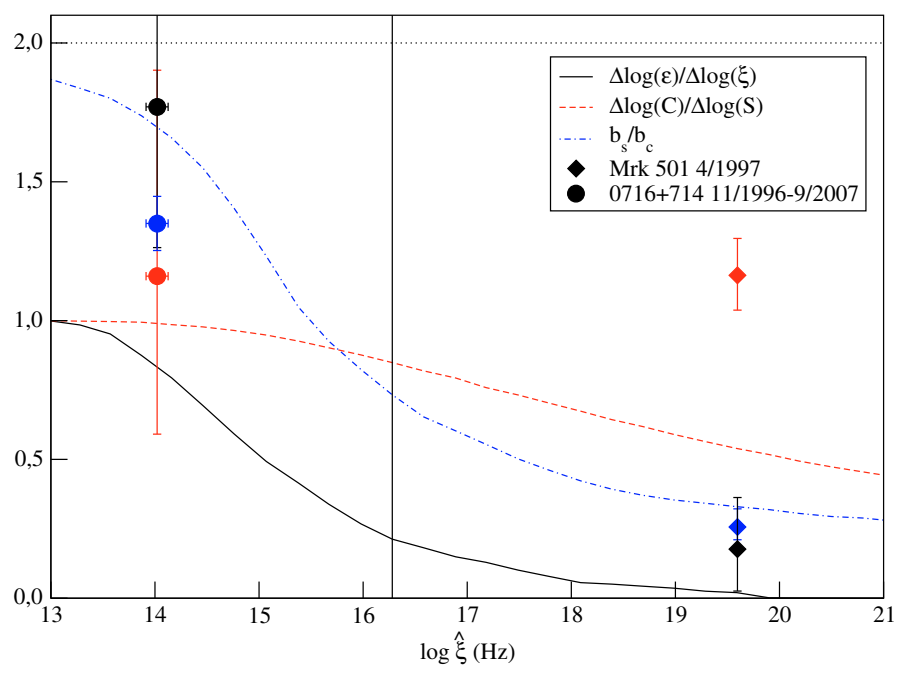

Fig. 5. Computed changes of spectral parameters as driven by an extended increase of the magnetic field with the other source parameters kept constant. The black full line represents the value of the ratio $\Delta \ln \epsilon / \Delta \ln \xi$, the red dashed line represents the value of the ratio $\Delta \ln C / \Delta \ln S$ and the blue dot-dashed line represents the ratio $b_{\mathrm{s}} / b_{\mathrm{c}}$, for different values of $\hat{\xi}=\xi\left(\gamma_{\mathrm{p}} / 10^{4}\right) 10^{(1-1 / 5 b) / 2}(\delta / 10)^{-1}(1+z)$ (where $\xi$ is the synchrotron SED peak frequency, see Eq. (15)), data points are the same as in Fig. 3; compared with the latter considerably larger changes of $n$ are needed to recover the observed values.

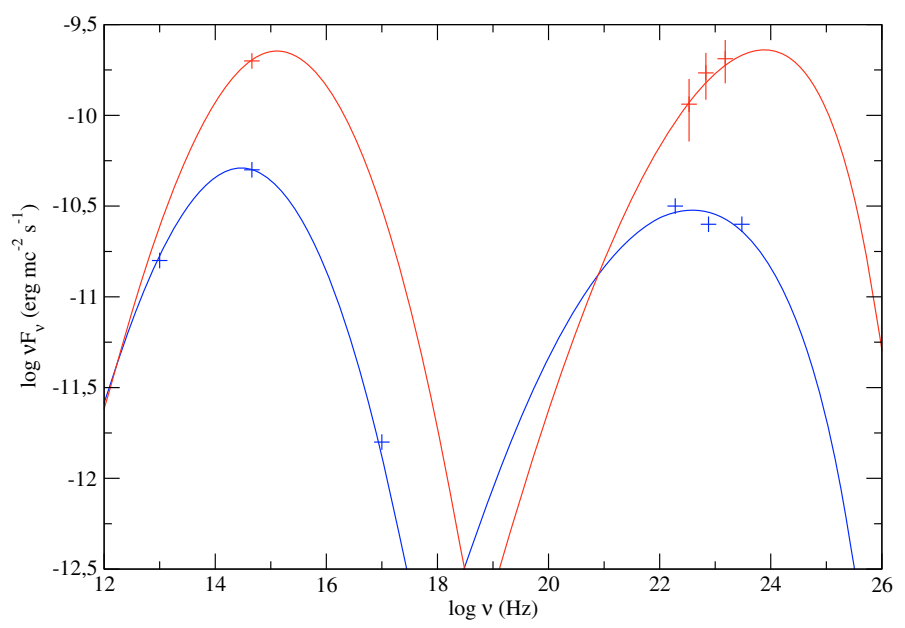

Fig. 6. Spectral fit of two different states of the IBL source $0716+714$. The low state (blue line) data are relative to 1996 November 14 simultaneous observation by AIT, BeppoSAX and EGRET; the high state (red line) data are relative to 2007 September 7-12 simultaneous observations by GASP-WEBT and AGILE-GRID (Tagliaferri et al. 2003; Chen et al. 2008).

around $\sigma_{\mathrm{T}} / 5$ (where $\sigma_{\mathrm{T}}$ is the Thomson cross section) when the $\gamma$-ray photon frequency $v$ and the target photons frequency $v_{\mathrm{t}}$ satisfy the relation $v_{\mathrm{t}} \approx m^{2} c^{4} \delta^{2} /\left[h^{2}(1+z)^{2} v\right]$.

For the $\gamma$ rays to escape from the emitting region the source "compactness" intervenes and can set lower bounds on the beaming factor (Cavaliere \& Morrison 1980; Massaro 2007; Begelman et al. 2008). In fact the optical depth for this process is expressed in terms of observational quantities (primed quantities refer to the jet rest frame) as

$\tau_{\gamma \gamma}\left(v_{\gamma}^{\prime}\right)=R \frac{\sigma_{\mathrm{T}}}{5} n_{\mathrm{ph}}^{\prime}\left(v_{\mathrm{t}}^{\prime}\right) \approx \frac{9}{20} \frac{\sigma_{\mathrm{T}} h}{m^{2} c^{6}} \frac{D^{2}}{\delta^{6}}(1+z)^{4} \frac{S v}{\Delta t}\left(\frac{v_{\mathrm{t}}}{\xi}\right)^{-\beta}$, where $D$ is the source distance expressed in Gpc, with the shorthand $\beta \equiv b \log \left(\frac{\nu_{\mathrm{t}}}{\xi}\right)$. Escaping $\gamma$-ray radiation requires the source to be optically thin, that is, $\tau_{\gamma \gamma}\left(v_{\gamma}^{\prime}\right) \leq 1$, resulting in

$\delta \geq\left[\frac{9}{20} \frac{\sigma_{\mathrm{T}} h^{1+2 \beta}}{m^{2+2 \beta} c^{6+4 \beta}} D^{2}(1+z)^{4+2 \beta} \frac{S v^{1+\beta} \xi^{\beta}}{\Delta t}\right]^{\frac{1}{6+2 \beta}}$.

Thus rapid flux variations set a lower bound on the beaming factor; the highest bound between the previous relation and $\delta \geqslant R(1+z) / c \Delta t$ is to be relevant.

The exponent $\beta$ contains a weak logarithmic dependence on $\delta$ that may be neglected to a first approximation, and has a magnitude which depends on the source spectral properties; for definiteness, we use for $b$ the typical value 0.2 , and for $v \approx 10^{27} \mathrm{~Hz}$. For a LBL with $\xi \sim 10^{14} \mathrm{~Hz}$ we obtain $\beta \approx$ 0.4 , while for an extreme HBL with $\xi \sim 10^{18} \mathrm{~Hz}$, we obtain $\beta \approx-0.5$. A variation timescale $\Delta t \sim 5$ min for a LBL with $S \sim 10^{-11} \mathrm{erg} \mathrm{cm}^{-2} \mathrm{~s}^{-1}$ implies $\delta \geqslant 30$, while in an extreme HBL with $S \sim 10^{-10} \mathrm{erg} \mathrm{cm}^{-2} \mathrm{~s}^{-1}$ we find $\delta \geqslant 15$.

In the specific case of PKS 2155-304 discussed by Begelman et al. (2008), the emitted power $L \approx 10^{46} \mathrm{erg} \mathrm{s}^{-1}$ corresponds to $S \sim 4 \times 10^{-11} \mathrm{erg} \mathrm{cm}^{-2} \mathrm{~s}^{-1}$, with $\xi \sim 10^{16} \mathrm{~Hz}$, to give $\beta \approx 0.1$ and $\delta \geqslant 50$ for $\Delta t \sim 5 \mathrm{~min}$. We note that the source, even if widely considered a HBL, shows peculiar behaviors with respect to other TeV HBLs (see Massaro et al. 2008b); moreover, it does not satisfy the condition $\xi \gg \xi_{\mathrm{T}}$ and so it cannot be considered an extreme HBL, as discussed below.

Extreme values of the beaming factor $\delta \sim 50$ have been proposed to account for large peak separations (Konopelko et al. 2003) or to explain very rapid spectral variations (Begelman et al. 2008), formally with $\Delta t \sim 5 \mathrm{~min}$ for the $\gamma$-ray flares of PKS 2155-304 in 2006 July 28 (Aharonian et al. 2007) and Mrk 501 in June 30 and July 9, 2007 (Albert et al. 2007a). Note that with BL Lac spectra realistically curved, the observed flux variations may be enhanced due to a slope effect best legible on the differential flux $F_{v}$; that is, when fluxes are measured at frequencies where the spectral slope is steep (as may be the case for PKS 2155-304), a strong observed flux variation implies only a mild variation of the peak flux and requires smaller values of $\delta$. Otherwise, a peak variation on a scale $\Delta t \sim 5 \mathrm{~min}$ would require in the SSC model $\delta \sim 100$ for LBLs where $\delta \propto \Delta t^{-\frac{1}{2}}$ (see Eq. (7)), and $\delta \sim 20$ for an extreme HBL where $\delta \propto \Delta t^{-\frac{2}{5}}$ (see Eq. (12)).

As stated under Sect. 3.3, in HBL sources even small flux variations in $\gamma$ rays should have enhanced counterparts in X rays. These may become hard to observe for example in a flare dominantly driven by particle number density increase associated with a magnetic field decrease (i.g., with $n \propto B^{-2}$ ), leading to a synchrotron emission easily drowned into other components. On the other hand, components with $\delta>20$ appearing in an HBL spectrum go beyond the HSZ SSC model with variations of one dominant parameter, and therefore require a more elaborate source structure.

In such cases the next natural scenarios are provided by decelerated relativistic outflows (Georganopoulos \& Kazanas 2003), or by nested spine-layer jets (Tavecchio \& Ghisellini 2008) and jets in a jet (Giannios et al. 2009).

\section{Beyond the SSC}

In Table 2 we have provided a comprehensive benchmark to gauge the performance of the HSZ SSC model for flaring BL Lac objects. This allows us to easily recognize events that may be 
accounted for within the model with physical variations of key source parameters, or that instead require more complex source structures.

Toward that purpose, we have used realistic log-parabolic spectral shapes produced by log-parabolic energy distributions of the emitting electrons, and studied IC radiation both in the Thomson and the Klein-Nishina regimes.

In the model we expect $S$ to dominate $C$ fluxes; in fact, moving to higher energies in a collection of different sources or in a prolonged evolution of a given source, we expect the emission to drift out of the pure Thomson and approach the KN regime, where the cross section decreases and limits the IC fluxes enforcing $C \lesssim S$. Such a relation is found to hold in a number of sources, and in particular in HBLs (see for example Tagliaferri et al. 2008).

From Table 2 we stress here the source spectral variations predicted in $\gamma$ rays. For LBLs, where the IC scattering mostly occurs in the Thomson regime, we recover the standard quadratic increase in $\gamma$-ray fluxes with respect to IR-optical ones, expressed by $\Delta \ln C \approx 2 \Delta \ln S$ for dominant particle rms energy variations (see Eq. (22)). Instead for HBLs, in which the IC scattering approaches the Klein-Nishina regime, we expect smaller or even vanishing increases of the $\gamma$-ray flux relative to X rays, that is, $\Delta \ln C \simeq 0$ for dominant particle rms energy variations (see Eq. (24)).

Comparing with specific sources, we find the HSZ SSC model to be adequate for most LBLs at the present observational levels; whilst for example the HBL source Mrk 501 in April 1997 showed a $\gamma$-ray flux increase appreciably stronger than expected if it were dominated by just one driving parameter. We explain this behavior within the model in terms of an additional, smooth and moderate increase (by a factor around 2) in number density of the electrons responsible for the emission (see Sect. 3.3). Such conditions can still be provided by a single electron population; this should be marked by a continuously decreasing spectral curvature $b$, as indicated by current data (Massaro et al. 2006), a feature providing a potentially powerful signature to closely check on further data. Instead, for dominant magnetic field variations we would expect $\Delta \ln C \approx \Delta \ln S$ for LBL sources (see Eq. (23)), and $\Delta \ln C \approx \frac{1}{2} \Delta \ln S$ for HBL sources (see Eq. 25)); but in that case the required increase in particle number density should be much higher by a factor of about 30, hard to interpret in terms of a single electron population.

On the other hand, on using the previous relations inversely, we see that even small $\gamma$-ray variations in HBL sources ought to have enhanced counterparts in the $\mathrm{X}$ rays, unless flare activity were driven by an additional jet component, for example one with higher magnetic field but lower particle density; thus the corresponding emission is easily overwhelmed by, or drowned into the main synchrotron, but then the rest frame acceleration times must be short enough to involve a significant fraction of the emitting region. We suggest this as a critical test for the simple model.

Another limitation arises from rapid peak flux variations requiring large values of $\delta$ as shown in Sect. 3.4. In the extreme, the few sources with particularly fast $\gamma$-ray increases observed so far, like PKS 2155-304 in 2006 July 28 and Mrk 501 in June 30 and July 9, 2007 (see Sect. 3.4), require additional components with very high beaming factors that go definitely beyond the simple SSC model.

Finally, another problem for the model arises from LBL objects showing substantial emissions in the GeV-TeV range that cannot be explained it terms of the simple HSZ SSC model (considering that second order IC scattering is negligible, see Massaro 2007) because the IC peak falls for these sources around $0.1 \mathrm{GeV}$; this may be the case for BL Lacertae itself (Albert et al. 2007b) and possibly similar sources like M87 (Georganopoulos et al. 2005) and Cen A (Lenian et al. 2008; Aharonian et al. 2009).

In all these cases the source may require more elaborate structure, like decelerated relativistic outflows or sub-jet scenarios (see Georganopoulos \& Kazanas 2003; Tavecchio \& Ghisellini 2008; Giannios et al. 2009). We stress that the injection of a second, monoenergetic electron population is expected to be marked by a sudden increase of the spectral curvature.

\section{Discussion and conclusions}

We propose that the sources of BL Lac type may be conveniently ordered in a succession spanning from smooth variations of one or a few dominant SSC parameter, up to the appearance of truly different components that ultimately break through the limits of the simple HSZ SSC model. Even more so at increasing energies and frequencies, that would imply weaker and weaker $\gamma$-ray fluxes relative to X-ray, owing to KN cross section effects. This picture is supported by the following two lines of evidence.

First, a recent statistical study of Third EGRET Catalogue data (Mukherjee et al. 2001; Casula 2008; Vagnetti et al., in prep.) shows for BL Lac objects observed at $30 \mathrm{MeV} \div 30 \mathrm{GeV}$ a weak $\gamma$-ray variability on average, compared to the FSRQ Blazars. Also the time-structure functions for these two classes of objects indicate a similar trend, within the limitations of the sample.

Second, the first AGILE-GRID Catalogue of high confidence gamma-ray sources (Pittori et al. 2009) apparently shows few if any new BL Lac sources other than those already detected by EGRET. This circumstance suggests the sources observed by AGILE to be more powerful than the average; it leads to expect for the majority of the sources either reduced flare activity in this band, or weak average fluxes. We relate these features to reduced Klein-Nishina cross section that tends to limit both the average fluxes and the flares.

With Fermi, the first three months of observations Abdo et al. (2009) have yielded a substantial number of new BL Lac sources, and in particular a larger fraction of HBLs close to the ratio known from radio surveys (Nieppola et al. 2006). This is consistent with our expectations, considering the better instrumental sensitivity, especially at high energies generally favorable to the HBLs; for these sources we suggest to test the model's outcomes: $C$ lower than $S$, and generally small (though possibly fast) flux variations compared to the LBLs

We plan to check our theoretical predictions against the public availability of the first year data from Fermi observations, and with simultaneous, multi-wavelength observations comparing the two basic subclasses of LBL and HBL of the BL Lac sources.

Acknowledgements. We thank our referee for useful comments and helpful suggestions. F. Massaro acknowledges the Foundation BLANCEFLOR Boncompagni-Ludovisi, née Bildt, for the grant awarded him in 2009 to support his research.

\section{References}

Abdo, A. A., Ackermann, M., Ajello, M., et al. 2009, [arXiv:0902 . 1559] Aharonian, F., Akhperjanian, A. G., Bazer-Bachi, A. R., et al. 2007, ApJ, 664, L71

Aharonian, F., Akhperjanian, A. G., Bazer-Bachi, A. R., et al. 2009, [arXiv: 0903.1582] 
Albert, J., Aliu, E., Anderhub, H., et al. 2007a, ApJ, 669, 862 Albert, J., Aliu, E., Anderhub, H., et al. 2007b. ApJ, 666, L17 Begelman, M. C., Fabian, A., \& Rees, M. J. 2008, MNRAS, 384, L19 Blandford, R. D., \& Znajek, R. L. 1977, MNRAS, 179, 433

Bloom, S. D., \& Marscher, A. P. 1996, ApJ, 461, 657

Blumenthal, G. R., \& Gould, R. J. 1970, Rev. Mod. Phys., 42, 237

Casula, V. 2008, Thesis

Cavaliere, A., \& D’Elia, V. 2002, ApJ, 571, 226

Cavaliere, A., \& Morrison, P. 1980, ApJ, 238, L63

Chen, A. W., D'Ammando, F., Villata, M., et al. 2008, A\&A, 489, L37

Djannati-Atai, A., Piron, F., Barrau, A., et al. 1999, A\&A, 350, 17

Donnarumma, I., Vittorini, V., Vercellone. S., et al. 2009, ApJ, 691, L13

Fossati, G., Buckley, J. H., Bond, I. H., et al. 2008, ApJ, 677, 906

Georganopoulos, M., \& Kazanas, D. 2003, ApJ, 594, 27

Georganopoulos, M., Perlman, E. S., \& Kazanas, D. 2005, ApJ, 634, L33

Giannios, D., Uzdensky, D. A., \& Begelman, M. C. 2009, [arXiv:0901.1877]

Giommi, P., Colafrancesco, S., Cutini, S., et al 2008, A\&A, 487, L49

Inoue, S., \& Takahara, F. 1996, ApJ, 463, 555

Jones, F. C. 1968, Phys. Rev., 167, 1159

Kaplan, S. A. 1956, Sov. Phys., 2, 2

Kardashev, N. S. 1962, SvA, 6, 317

Kataoka, J., Mattox, J. R., Quinn, J., et al. 1999, ApJ, 514, 138

Katarzyński, K., Ghisellini, G., Tavecchio, F., et al. 2005, A\&A, 433, 479

Konopelko, A., Mastichiadis, A., Kirk, J., et al. 2003, ApJ, 597, 851

Landau, R., Golisch, B., Jones, T., et al. 1986, ApJ, 308, 78

Lapi, A., Cavaliere, A., \& Menci, N. 2005, ApJ, 619, 90

Lenain, J.-P., Boisson, C., \& Sol, H. 2008, [arXiv: 0807.2733]

Maraschi, L., Ghisellini, G., \& Celotti, A. 1992, ApJ, 397, L5

Marscher, A. P. 1996, Energy Transport in Radio Galaxies and Quasars, ASP Conf. Ser., 100, 45
Marscher, A. P., \& Gear, W. K. 1985, ApJ, 298, 114

Massaro, F. 2007, Ph.D. Thesis

Massaro, E., Perri, M., Giommi, P., \& Nesci, R. 2004a, A\&A, 413, 489

Massaro, E., Perri, M., Giommi, P., Nesci, R., \& Verrecchia, F. 2004b, A\&A, 422, 103

Massaro, E., Tramacere, A., Perri, M., Giommi, P., \& Tosti, G. 2006, A\&A, 448, 861

Massaro, F., Tramacere, A., Cavaliere, A., Perri, M., \& Giommi, P. 2008a, A\&A, 478, 395

Massaro, F., Giommi, P., Tosti, G., et al. 2008b, A\&A, 489, 1047

Mukherjee, R., et al. 2001, in High Energy Gamma-Ray Astronomy, ed. F. A. Aharonian, \& H. J. Völk (New York: AIP), AIP Conf. Proc., 558, 324

Nieppola, E., Tornikoski, M., \& Valtaoja, E. 2006, A\&A, 445, 441

Ostriker, J. P., \& McKee, C. F. 1988, Rev. Mod. Phys., 61, 1

Padovani, P., \& Giommi, P. 1995, ApJ, 444, 567

Paggi, A. 2007, Thesis

Pittori, C., Verrecchia, F., Chen, A. W., et al. 2009, [arXiv: 0902 . 2959]

Rybicki, G. B., \& Lightman, A. P. 1979, Radiative Processes in Astrophysics (New York: Wiley)

Sikora, M., Begelman, M. C., \& Rees, M. J. 1994, ApJ, 421, 123

Tagliaferri, G., Ravasio, M., \& Ghisellini, G. 2003, A\&A, 400, 477

Tagliaferri, G., Foschini, L., Ghisellini, G., et al. 2008, ApJ, 679, 1029

Tanihata, C., Kataoka, J., Takahashi, T., et al. 2004, ApJ, 601, 759

Tavecchio, F., \& Ghisellini, G. 2008, [arXiv:0810.0134]

Tavecchio, F., Maraschi, L., \& Ghisellini, G. 1998, ApJ, 509, 608

Tramacere, A., Massaro, F., \& Cavaliere, A. 2007, A\&A, 466, 521

Tramacere, A., Giommi, P., Perri, M., Verrecchia, F., \& Tosti, G. 2009, [arXiv:0901.4124v1]

Vietri, M. 2006, Astrofisica delle alte energie, Torino, Bollati Boringhieri 


\section{Appendix A: Log-parabolic spectra}

In this Appendix we show how electron populations with a logparabolic energy distribution of the form expressed by Eq. (3), that is,

$N(\gamma)=N_{0}\left(\frac{\gamma}{\gamma_{0}}\right)^{-s-r \log \left(\frac{\gamma}{\gamma_{0}}\right)}$,

emit log-parabolic spectra via the SSC process. The related particle synchrotron emissivity

$j_{v}^{\mathrm{s}}=\int \mathrm{d} \gamma N(\gamma) \frac{\mathrm{d} P_{\mathrm{s}}}{\mathrm{d} v}$

is easily computed on using the close approximation to the single particle emission in the shape of a delta-function (see Rybicki \& Lightmann 1979), that is, $\frac{\mathrm{d} P_{\mathrm{s}}}{\mathrm{d} v} \approx P_{\mathrm{s}} \delta\left(v-\gamma^{2} v_{\mathrm{c}}\right)$ with $P_{\mathrm{s}}=1 / 6 \pi \sigma_{\mathrm{T}} \gamma^{2} c B^{2}$ and $v_{\mathrm{c}} \approx 1.22 \times 10^{6} B \mathrm{~Hz}$ (with $B$ measured in Gauss) is the synchrotron critical frequency. This leads to (Massaro et al. 2004a) a log-parabolic differential flux

$F_{v}^{\mathrm{s}} \approx F_{0}\left(\frac{v}{v_{0}}\right)^{-a_{\mathrm{s}}-b_{\mathrm{s}} \log \left(\frac{v}{v_{0}}\right)}$,

and to a SED again of log-parabolic shape

$S_{v}^{\mathrm{s}}=v F_{v}^{\mathrm{s}} \approx S_{0}^{\mathrm{s}}\left(\frac{v}{v_{0}}\right)^{-\left(a_{\mathrm{s}}-1\right)-b_{\mathrm{s}} \log \left(\frac{v}{v_{0}}\right)} ;$

its slope at the synchrotron reference frequency $v_{0}$ is given in terms of $s$, by

$a_{\mathrm{s}} \approx \frac{s-1}{2}$,

the spectral curvature by

$b_{\mathrm{s}} \approx \frac{r}{4}$,

and the peak value $S^{\prime} \propto R^{3} B^{2} n \gamma_{\mathrm{p}}^{2} \sqrt{r}$ occurs at a frequency $\xi^{\prime} \propto B \gamma_{\mathrm{p}}^{2} \times 10^{\frac{1}{r}}\left(\gamma_{\mathrm{p}}, n, B\right.$ and $R$ are defined in Sect. 2.1 of the main text).

For IC radiation in the Thomson regime we may write to a fair approximation $\frac{\mathrm{d} P_{\mathrm{c}}}{\mathrm{d} v} \approx P_{\mathrm{c}} \delta\left(v-\frac{4}{3} \gamma^{2} \xi^{\prime}\right)$ (see Rybicky \& Lightmann 1979) where $P_{\mathrm{c}}=\frac{4}{3} \sigma_{\mathrm{T}} \gamma^{2} c \epsilon_{v}$ is the power radiated by a single-particle IC scattering in the Thomson regime, having denoted with $\epsilon_{v} \propto R B^{2} n \gamma_{\mathrm{p}}^{2}$ the synchrotron radiation density ${ }^{2}$. We obtain once again a log-parabolic SED

$S_{v}^{\mathrm{c}} \approx S_{0}^{\mathrm{c}}\left(\frac{v}{\hat{v}_{0}}\right)^{-\left(a_{\mathrm{c}}-1\right)-b_{\mathrm{c}} \log \left(v / \hat{v}_{0}\right)}$,

where the slope at the IC reference frequency $\hat{v}_{0}$ is given by

$a_{\mathrm{c}} \approx \frac{s-1}{2}$,

and the spectral curvature reads

$b_{\mathrm{c}} \approx \frac{r}{4}$.

2 Note that in Thomson regime no direct relation appears between electrons emitting peak synchrotron photons and those responsible of IC peak radiation (see Tavecchio et al. 1998).
The peak value $C^{\prime} \propto R^{4} B^{2} n^{2} \gamma_{\mathrm{p}}^{4} \sqrt{r}$ is attained at a frequency $\epsilon^{\prime} \propto B \gamma_{\mathrm{p}}^{4}$.

For the Klein-Nishina $(\mathrm{KN})$ regime instead it necessary to consider the convolution

$j_{v}^{\mathrm{c}}=h \int \mathrm{d} \gamma N(\gamma) \int \mathrm{d} \tilde{v} v N_{\tilde{v}} K(v, \tilde{v}, \gamma)$

where $\tilde{v}$ and $v$ are the electron frequencies before and after the scattering, respectively, $N_{\tilde{v}}$ is the number spectrum of seed photons, and $K(v, \tilde{v}, \gamma)$ is the full Compton kernel (Jones 1968). Only in the extreme $\mathrm{KN}$ regime one may again approximate

$K(v, \tilde{v}, \gamma) \approx \frac{1}{\gamma^{2}} \delta\left(1-\frac{h v}{\gamma m c^{2}}\right)$

on approximating $N_{\tilde{v}}$ with its mean value for a homogeneous, spherical optically thin source

$N_{\tilde{v}} \approx\left\langle N_{\tilde{v}}\right\rangle=\frac{3}{4} \frac{R}{c} \frac{j_{\tilde{v}}^{\mathrm{s}}}{h \tilde{v}}$,

one obtains again a SED with a log-parabolic shape

$S_{v}^{\mathrm{c}} \approx S_{0}^{\mathrm{c}}\left(\frac{v}{\hat{v}_{0}}\right)^{-\left(a_{\mathrm{c}}-1\right)-b_{\mathrm{c}} \log \left(v / \hat{v}_{0}\right)}$.

Now the slope at $\hat{v}_{0}$

$a_{\mathrm{c}} \approx s$,

is steeper, and the spectral curvature

$b_{\mathrm{c}} \approx r$

is larger than in the Thomson regime. The peak value $C^{\prime} \propto$ $R^{4} B n^{2} \sqrt{r}$ occurs at a frequency $\epsilon^{\prime} \propto \gamma_{\mathrm{p}} \times 10^{-\frac{1}{2 r}}$.

The transition between the two regimes occurs when $2 \gamma_{\max } h \xi^{\prime} \approx m c^{2}$, where $\gamma_{\max }=\sqrt{\xi^{\prime} / \nu_{\mathrm{c}}}$, that is, when

$\xi_{\mathrm{T}} \approx 7.15 \times 10^{15}\left(\frac{B}{0.1 \mathrm{G}}\right)^{\frac{1}{3}}\left(\frac{\delta}{10}\right)(1+z)^{-1} \mathrm{~Hz}$

holds, or equivalently

$\xi_{\mathrm{T}} \approx 1.96 \times 10^{16}\left(\frac{\gamma_{\mathrm{p}}}{10^{4}}\right)^{-1} 10^{\frac{1}{2}\left(1-\frac{1}{5 b}\right)}\left(\frac{\delta}{10}\right)(1+z)^{-1} \mathrm{~Hz}$.

We close these calculations with two remarks. First, the (primed) quantities used here refer to the rest frame of the emitting region, while the observed (unprimed) quantities must be multiplied by powers of the beaming factor

$\delta=\frac{1}{\Gamma(1-\beta \cos \theta)}$,

$\Gamma$ being the bulk Lorentz factor of the relativistic electron flow in the emitting region, $\theta$ the angle between its velocity $v$ and the line of sight, and $\beta=v / c$; so we obtain $S=S^{\prime} \delta^{4}, C=C^{\prime} \delta^{4}$, and $\xi=\xi^{\prime} \delta, \epsilon=\epsilon^{\prime} \delta$. Second, we note that the actual specific powers $\frac{\mathrm{d} P}{\mathrm{~d} v}$ radiated by a single particle differ from delta-function shape, slightly for the synchrotron emission and considerably for the IC radiation in the Thomson regime (see discussion in Rybicky \& Lightmann 1979). However, the above spectral shapes still approximatively apply, as confirmed by numerical simulations (Massaro 2007); in detail, the convolution with a broader single particle power yields a less curved spectrum, so that Eqs. (A.6) and (A.9) become $b_{\mathrm{s}} \approx r / 5$ (Massaro et al. 2006) and $b_{\mathrm{c}} \approx r / 10$, respectively. 


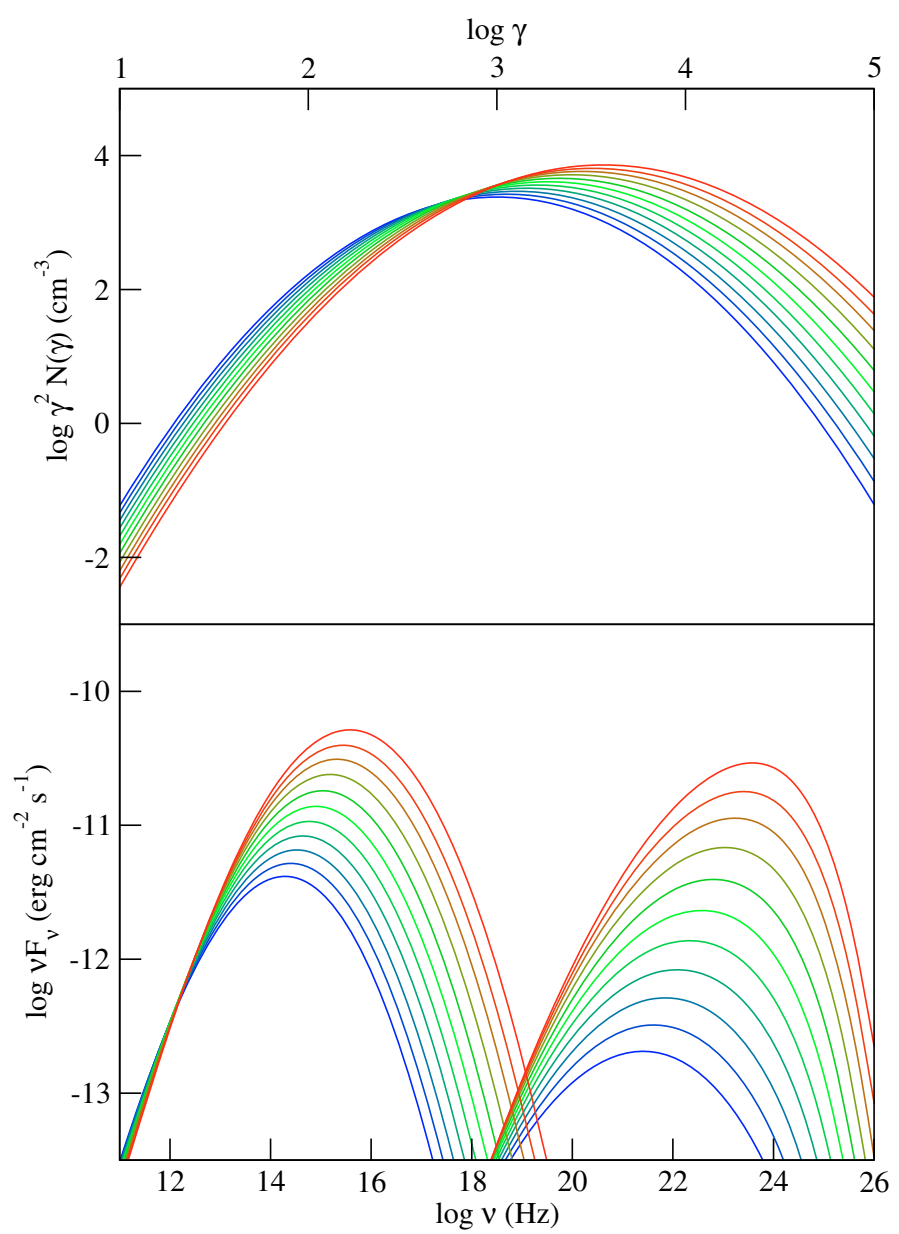

Fig. B.1. Example of time evolution of the $\gamma^{2} N(\gamma)$ distribution (with peak energy $\gamma_{\mathrm{p}}$ ) due to stochastic and systematic accelerations (upper panel) with $\lambda_{1}=5 \times 10^{-3}$ days $^{-1}, \lambda_{2}=5 \times 10^{-4}$ days $^{-1}$ from the initial value $\gamma_{\mathrm{p}}=10^{3}$, and of the related evolution for the SSC SEDs (lower panel). In terms of the stochastic acceleration time $\tau_{2}$, the time interval between each pair of lines is $t_{2}-t_{1}=10^{-2} \tau_{2}$, corresponding to an observed time interval of about two days.

\section{Appendix B: Particle acceleration processes}

Here we derive a log-parabolic electron energy distribution $N(\gamma, t)$ from a kinetic continuity equation of the Fokker-Planck type; following Kardashev (1962), in the jet rest frame this reads

$$
\frac{\partial N}{\partial t}=-\lambda_{1}(t) \frac{\partial}{\partial \gamma}(\gamma N)+\lambda_{2}(t) \frac{\partial}{\partial \gamma}\left(\gamma^{2} \frac{\partial N}{\partial \gamma}\right)
$$

where $\gamma m c^{2}$ is the particle energy, $t$ denotes time, and $\lambda_{1}$ and $\lambda_{2}$ describe systematic and stochastic acceleration rates, occurring on timescales $t_{1}=1 / \lambda_{1}$ and $t_{2}=1 / \lambda_{2}$, respectively. For example, in the picture of Fermi accelerations (e.g., Vietri 2006), the accelerations rates $\lambda_{1}$ and $\lambda_{2}$ can be expressed in terms of physical quantities related to processes occurring in shocks; in this framework a plane shock front of thickness $\ell_{\mathrm{s}}$ moves with speed $V_{\mathrm{s}}$ and gas clouds of average size $\ell$ move downstream of the shock with speed $V$; it is found that $\lambda_{1}=V_{\mathrm{s}} / \ell_{\mathrm{s}}$ and $\lambda_{2}=V^{2} / 2 c \ell$ hold (see Kaplan 1956; Paggi 2007), $\lambda_{2} \ll \lambda_{1}$. On the other hand, numerical values of $\lambda_{1}$ and $\lambda_{2}$ are directly derived from the emitted spectrum as shown later.

The Fokker-Planck Eq. (B.1) describes the evolution of the electron distribution function; with an initially mono-energetic distribution in the form of a delta-function $N(\gamma, 0)=n \delta\left(\gamma-\gamma_{0}\right)$

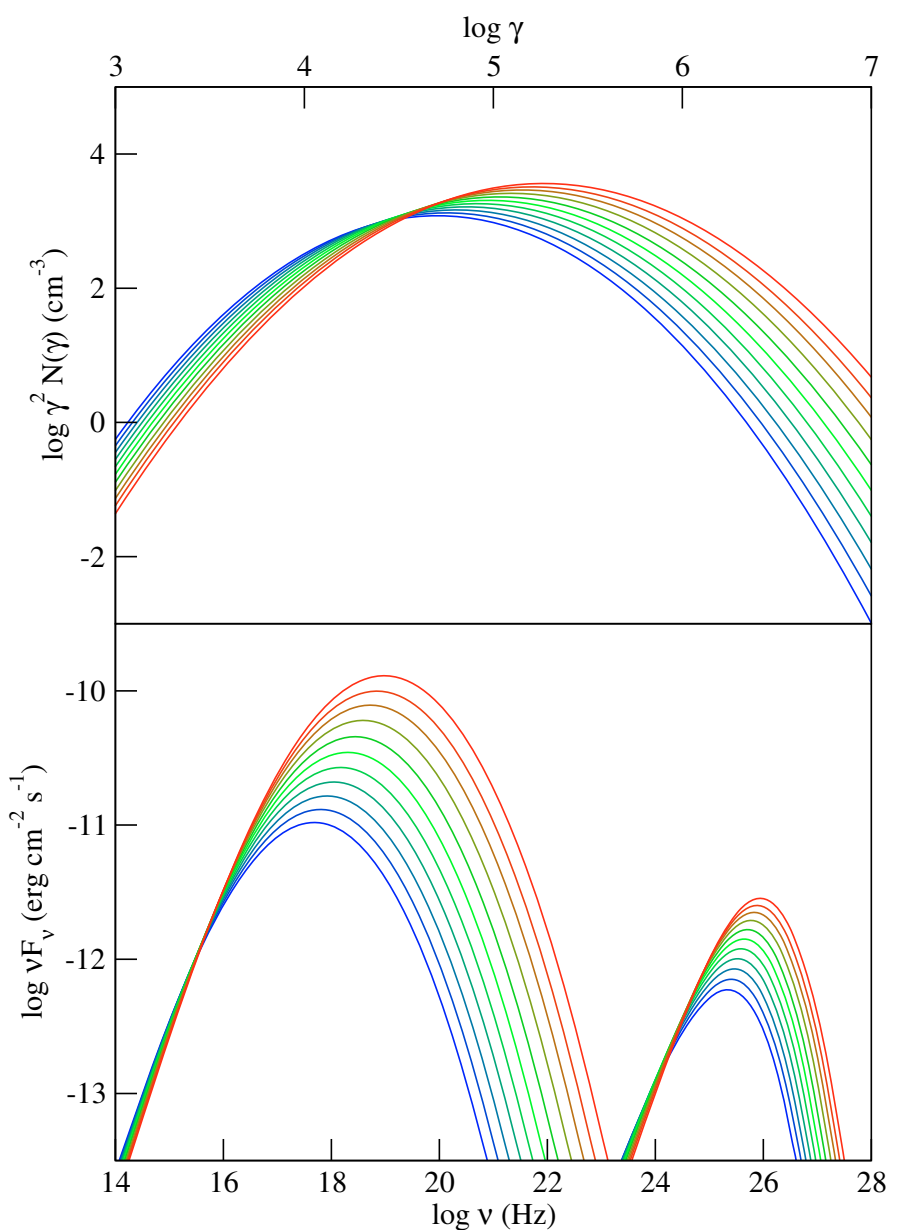

Fig. B.2. Example of time evolution of the $\gamma^{2} N(\gamma)$ distribution (with peak energy $\gamma_{\mathrm{p}}$ ) due to stochastic and systematic accelerations (upper panel) with $\lambda_{1}=5 \times 10^{-3}$ days $^{-1}, \lambda_{2}=5 \times 10^{-4}$ days $^{-1}$ from the initial value $\gamma_{\mathrm{p}}=5 \times 10^{4}$, and of the related evolution for the SSC SEDs (lower panel). In terms of the stochastic acceleration time $\tau_{2}$, the time interval between each pair of lines is $t_{2}-t_{1}=10^{-2} \tau_{2}$, corresponding to an observed time interval of about two days.

( $n$ is the initial particle number density) the solution at subsequent times $t$ takes the form of the log-parabolic energy distribution assumed in Eq. (3) of the main text (see also A.1), reading

$N(\gamma, t)=N_{0}\left(\frac{\gamma}{\gamma_{0}}\right)^{-s-r \log \left(\frac{\gamma}{\gamma_{0}}\right)}$.

Here the time depending slope at $\gamma=\gamma_{0}$ is given by

$s=\frac{1}{2}\left(1-\frac{\int \mathrm{d} t \lambda_{1}}{\int \mathrm{d} t \lambda_{2}}\right)$

meanwhile, the curvature of $N(\gamma)$ driven by the diffusive (stochastic) term in Eq. (B.1), irreversibly decreases in time after

$r=\frac{\ln 10}{4 \int \mathrm{d} t \lambda_{2}}$

from the large initial values corresponding to the initially monoenergetic distribution. Correspondingly, the time-dependent height at $\gamma=\gamma_{0}$ follows

$N_{0}=\frac{1}{2 \sqrt{\pi}} \frac{n}{\gamma_{0}} \frac{1}{\sqrt{\int \mathrm{d} t \lambda_{2}}} \exp \left[-\frac{\left(\int \mathrm{d} t \lambda_{1}+\int \mathrm{d} t \lambda_{2}\right)^{2}}{4 \int \mathrm{d} t \lambda_{2}}\right]$ 
To wit, Eq. (B.2) describes the evolution of the electron distribution, growing broader and broader under the effect of stochastic acceleration, while its peak moves from $\gamma_{0}$ to the current position

$\gamma_{M}=\gamma_{0} \mathrm{e}^{\int \mathrm{d} t\left(\lambda_{1}-\lambda_{2}\right)}$

under the contrasting actions of the systematic and stochastic accelerations. An important quantity to focus on for the emission properties is the rms energy

$$
\begin{aligned}
\gamma_{\mathrm{p}} & \equiv \sqrt{\frac{\int \gamma^{2} N(\gamma) \mathrm{d} \gamma}{\int N(\gamma) \mathrm{d} \gamma}}=\gamma_{0} \mathrm{e}^{\int\left(\lambda_{1}+3 \lambda_{2}\right) \mathrm{d} t} \\
& =\gamma_{0} 10^{\frac{2-s}{2 r}}=\gamma_{M} \mathrm{e}^{4 \int \lambda_{2} \mathrm{~d} t},
\end{aligned}
$$

which is also the position for the peak of the distribution $\gamma^{2} N(\gamma)$.

We have already derived in Appendix A the shapes of the spectra (synchrotron, and IC in both the Thomson and $\mathrm{KN}$ regimes) emitted by the distribution given in Eq. (3); here we stress the time dependence of their main spectral features. We can write for the synchrotron emission ${ }^{3}$

$S \propto \frac{\mathrm{e}^{2 \int\left(\lambda_{1}+3 \lambda_{2}\right) \mathrm{d} t}}{\sqrt{\int \lambda_{2} \mathrm{~d} t}} \propto \gamma_{\mathrm{p}}^{2} \sqrt{r}, \quad \xi \propto \mathrm{e}^{2 \int\left(\lambda_{1}+5 \lambda_{2}\right) \mathrm{d} t} \propto \gamma_{\mathrm{p}}^{2} \times 10^{\frac{1}{r}} ;$

for IC emission we have in the Thomson regime

$C \propto \frac{\mathrm{e}^{4 \int\left(\lambda_{1}+3 \lambda_{2}\right) \mathrm{d} t}}{\sqrt{\int \lambda_{2} \mathrm{~d} t}} \propto \gamma_{\mathrm{p}}^{4} \sqrt{r}, \quad \epsilon \propto \mathrm{e}^{4 \int\left(\lambda_{1}+5 \lambda_{2}\right) \mathrm{d} t} \propto \gamma_{\mathrm{p}}^{4}$,

and in the extreme $\mathrm{KN}$ regime

$C \propto \frac{1}{\sqrt{\int \lambda_{2} \mathrm{~d} t}} \propto \sqrt{r}, \quad \epsilon \propto \mathrm{e}^{\int\left(\lambda_{1}+\lambda_{2}\right) \mathrm{d} t} \propto \gamma_{\mathrm{p}} \times 10^{-\frac{1}{2 r}}$.

Note that during flares, since $\lambda_{1} \gg \lambda_{2}$, we expect the curvature to vary so little that we can therefore approximatively write $S \propto \xi$ (see main text).

Now we focus the above relations for the simple case of time independent $\lambda_{1}$ and $\lambda_{2}$, when $\int \lambda_{1,2} \mathrm{~d} t \approx \lambda_{1,2} t$; then we have

$s=\frac{1}{2}\left(1-\frac{\lambda_{1}}{\lambda_{2}}\right), \quad r=\frac{\ln 10}{4 \lambda_{2} t} \approx \frac{0.58}{\lambda_{2} t}$,

while for the rms energy we have

$\gamma_{\mathrm{p}}=\gamma_{0} e^{\left(\lambda_{1}+3 \lambda_{2}\right) t}$

so for the peak frequency and the spectral curvature we have

$\log \left(\frac{\xi}{\xi_{0}}\right)=2\left(\lambda_{1}+5 \lambda_{2}\right) t, \quad b_{\mathrm{s}}=\frac{1}{10}\left(5+\frac{\lambda_{1}}{\lambda_{2}}\right) \frac{1}{\log \left(\frac{\xi}{\xi_{0}}\right)}$

where $\xi_{0}$ is a normalization frequency. It is seen that soon after the injection the curvature $b_{\mathrm{s}}$ (proportional to $r$ ) drops rapidly, then progressively decreases more and more gently, while the peak frequency still increases.

The value of $\lambda_{2}$ can be evaluated from observing the synchrotron spectral curvatures $b_{2}$ and $b_{1}$ at two times $t_{2}$ and $t_{1}$,

\footnotetext{
${ }^{3}$ Note that singular behaviors of $S$ and $C$ for $t=0$ are a consequence of the (differential) definition of the SED as $v F_{v}$, and relate to the initial singularity of the particle energy distribution. Integrated quantities like $F=\int \mathrm{d} v F_{v}$ behave regularly.
}

respectively (recall that $b_{\mathrm{s}} \approx r / 5$ ); denoting with $t_{2}-t_{1}$ this time interval, we have

$\lambda_{2}=\frac{0.58}{t_{2}-t_{1}}\left(\frac{1}{r_{2}}-\frac{1}{r_{1}}\right) \frac{1+z}{\delta}$

on the other hand, form observing the related synchrotron peaks $\xi_{2}$ and $\xi_{1}$, the value of $\lambda_{1}$ can be evaluated as

$\lambda_{1}=\left[\frac{1}{2\left(t_{2}-t_{1}\right)} \ln \left(\frac{\xi_{2}}{\xi_{1}}\right)-\frac{2.88}{t_{2}-t_{1}}\left(\frac{1}{r_{2}}-\frac{1}{r_{1}}\right)\right] \frac{1+z}{\delta}$.

For example, in the case of Mrk 501 in the states of 7 and 16 April 1997, we obtain (on assuming $B \approx$ const.) $\lambda_{1}=$ (2.3 \pm 1.1$) \mathrm{yr}^{-1}$ and $\lambda_{2}=(1.8 \pm 1.7) 10^{-1} \mathrm{yr}^{-1}$, corresponding to acceleration times $\tau_{1}=1 / \lambda_{1}=(4.3 \pm 2.0) 10^{-1} \mathrm{yr}$ and $\tau_{2}=1 / \lambda_{2}=(5.5 \pm 5.0) \mathrm{yr}$. Note that with the current data the evaluations of $\lambda_{1}$ and $\lambda_{2}$ turn out to be affected by uncertainties considerably larger than the single curvatures $b_{1}=0.161 \pm 0.007$ and $b_{2}=0.148 \pm 0.005$.

If the total energy available to the jet is limited (e.g., by the BZ limit, see text) we expect that $\int \mathrm{d} t \lambda_{1}$ and $\int \mathrm{d} t \lambda_{2}$ cannot grow indefinitely, but are to attain a limiting value. At low energies where $\int \mathrm{d} t \lambda_{1} \gg \int \mathrm{d} t \lambda_{2}$ holds, we have $S \propto \xi$ and $C \propto \epsilon$ as before; at higher energies when $\int \mathrm{d} t \lambda_{1}$ reaches its limit, $\int \mathrm{d} t \lambda_{1} \ll \int d t \lambda_{2}$ holds, leading to $S \propto \xi^{0.6}$ and $C \propto \epsilon^{0.6}$. Eventually also $\int \mathrm{d} t \lambda_{2}$ reaches its limit, and both the fluxes and the peak frequencies cannot grow any more.

\section{Appendix C: Prefactors for Eqs. (4)-(13)}

The HSZ SSC model is, as stated before, characterized by five parameters: the rms particle energy $\gamma_{\mathrm{p}}$, the particle density $n$, the magnetic field $B$, the size of the emitting region $R$ and the beaming factor $\delta$. So the model may be constrained by five observables that we denote with

$S_{i} \equiv \frac{S}{10^{i} \frac{\mathrm{erg}}{\mathrm{cm}^{2} \mathrm{~s}}}, \quad C_{j} \equiv \frac{C}{10^{j} \frac{\mathrm{erg}}{\mathrm{cm}^{2} \mathrm{~s}}}$,

$\xi_{k} \equiv \frac{\xi}{10^{k} \mathrm{~Hz}}, \quad \epsilon_{h} \equiv \frac{\epsilon}{10^{h} \mathrm{~Hz}}$

where the indexes $i, j, k, h$ express the normalizations demonstrated below; in addition, we denote with $\Delta t_{\mathrm{d}}$ the time in days for the source variations, and with $D$ the distance of the source in Gpc.

In the Thomson regime we find

$$
\begin{aligned}
& B=1.04 \times 10^{-1} \\
& \times\left[b^{\frac{1}{8}} D^{-\frac{1}{2}}(1+z)^{\frac{1}{2}} \xi_{14}{ }^{3} C_{-11^{\frac{1}{4}}} \Delta t_{\mathrm{d}}^{\frac{1}{2}} \epsilon_{22}{ }^{-\frac{3}{2}} S_{-11}{ }^{-\frac{1}{2}}\right] \mathrm{G} \\
& \delta=13.5 \\
& \times\left[D^{\frac{1}{2}} b^{-\frac{1}{8}}(1+z)^{\frac{1}{2}} \epsilon_{22^{\frac{1}{2}}} S_{-11^{\frac{1}{2}}} \xi_{14}{ }^{-1} C_{-11}{ }^{-\frac{1}{4}} \Delta t_{\mathrm{d}}{ }^{-\frac{1}{2}}\right] \\
& R=3.50 \times 10^{16}
\end{aligned}
$$

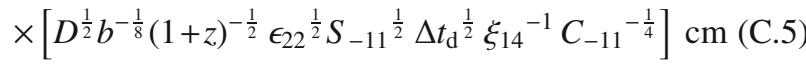

$$
\begin{aligned}
& n=5.74 \times\left[b^{\frac{1}{8}} D^{\frac{1}{2}}(1+z)^{\frac{1}{2}} \times 10^{\left(\frac{1}{5 b}-1\right)} \xi_{14}{ }^{2}\right. \\
& \left.\times C_{-11^{\frac{5}{4}}} \epsilon_{22}{ }^{-\frac{3}{2}} S_{-11}{ }^{-\frac{3}{2}} \Delta t_{\mathrm{d}}{ }^{-\frac{1}{2}}\right] \mathrm{cm}^{-3} \\
& \gamma_{\mathrm{p}}=2.74 \times 10^{3}\left[10^{\frac{1}{2}\left(1-\frac{1}{5 b}\right)} \epsilon_{22^{\frac{1}{2}}} \xi_{14^{-\frac{1}{2}}}\right] \text {. }
\end{aligned}
$$


A. Paggi et al.: SSC radiation in BL Lacertae sources, the end of the tether, Online Material p 4

For the extreme $\mathrm{KN}$ regime we obtain

$$
\begin{aligned}
& B=1.72 \times 10^{-2} \\
& \times\left[D^{\frac{2}{5}} b^{\frac{1}{10}}(1+z)^{-\frac{2}{5}} \xi_{18^{\frac{2}{5}}} S_{-10^{\frac{2}{5}}} \epsilon_{26^{-\frac{8}{5}}} C_{-11}{ }^{-\frac{1}{5}} \Delta t_{\mathrm{d}}{ }^{-\frac{2}{5}}\right] \mathrm{G} \quad \text { (C.8) } \\
& \delta=4.21 \times\left[D^{\frac{2}{5}} b^{-\frac{1}{10}} 10^{\frac{4}{5}\left(\frac{1}{5 b}-1\right)}(1+z)^{\frac{3}{5}} \epsilon_{26^{\frac{2}{5}}}\right. \\
& \left.\times S_{-10^{\frac{2}{5}}} \xi_{18}{ }^{-\frac{3}{5}} C_{-11}{ }^{-\frac{1}{5}} \Delta t_{\mathrm{d}}{ }^{-\frac{2}{5}}\right] \\
& R=1.0910^{16} \times\left[D^{\frac{2}{5}} b^{-\frac{1}{10}} \times 10^{\frac{4}{5}\left(\frac{1}{5 b}-1\right)}(1+z)^{-\frac{2}{5}} \epsilon_{26^{\frac{2}{5}}}\right.
\end{aligned}
$$

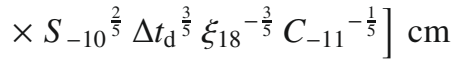

$$
\begin{aligned}
& n=952 \times\left[b^{\frac{1}{5}} D^{-\frac{4}{5}} \times 10^{\frac{13}{5}\left(1-\frac{1}{5 b}\right)}(1+z)^{\frac{4}{5}} \xi_{18^{\frac{11}{5}}} C_{-11^{\frac{7}{5}}} \epsilon_{26^{-\frac{4}{5}}}\right. \\
& \left.\times S_{-10}{ }^{-\frac{9}{5}} \Delta t_{\mathrm{d}}{ }^{-\frac{1}{5}}\right] \mathrm{cm}^{-3} \\
& \gamma_{\mathrm{p}}=6.07 \times 10^{5} \times\left[b^{\frac{1}{10}} D^{-\frac{2}{5}} \times 10^{\frac{3}{10}\left(1-\frac{1}{5 b}\right)}(1+z)^{\frac{2}{5}} \xi_{18^{\frac{3}{5}}} \epsilon_{26^{\frac{3}{5}}}\right.
\end{aligned}
$$

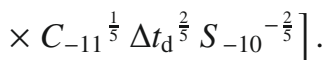

